\title{
Relevância da biomecânica da córnea no glaucoma
}

\section{Relevancy of corneal biomechanical in glaucoma}

Jorge Augusto Siqueira da Silva ${ }^{1}$, Renata Siqueira da Silva ${ }^{2,3}$, Renato Ambrósio Jr ${ }^{2,3}$

\begin{abstract}
Resumo
Relato de caso de uma paciente, com córnea espessa, por distrofia de Fuchs em fase inicial, ainda com transparência corneana preservada (edema corneano subclínico), associado à pressão intraocular (PIO) normal por tonometria de aplanação de Goldman (TAG), que teve entretanto, o diagnóstico de glaucoma primário de ângulo aberto com base em alterações estruturais do nervo óptico. O estudo tomográfico demonstrou padrão de paquimetria espacial compatível com edema sub-clínico. O estudo biomecânico da córnea com o ORA (Ocular Response Analyzer, ( Reichert), associado à medida corrigida da PIO, possibilitou o entendimento da influência da córnea, que apesar de mais espessa, levava a uma TAG falsamente reduzida (hipoestimada). O estudo da PIO com sistema de tonografia digital de contorno (PASCAL) corroborou com os achados do ORA. Este exemplo ressalta a importância de novas tecnologias na avaliação de pacientes com suspeita de glaucoma, e destaca que a correção da TAG, com base em algoritmos lineares relacionados com a paquimetria central apenas, pode determinar sérios erros de interpretação clínica.
\end{abstract}

Descritores: Córnea/fisiologia; Glaucoma; ORA; Distrofia endotelial de Fuchs; Tonometria ocular/métodos; Relatos de casos

\section{Abstract}

Case report of a patient, with a thick cornea, for Fuchs dystrophy in its early stages, yet with preserved corneal transparency (subclinical corneal edema), associated with normal intraocular pressure (IOP) by Goldman applanation tonometry (GAT), which was however, the diagnosis of primary open-angle glaucoma on the basis of structural changes of the optic nerve. The tomographic study showed a pattern of spatial pachymetry compatible with subclinical edema. The corneal biomechanical study with ORA (Ocular Response Analyzer, Reichert ${ }^{\circledR}$ ) associated with corrected IOP measurement, allowed the understanding of the corneal influence, which, although thicker, leading to a falsely low TAG (underestimated). The IOP study with tonography system digital (PASCAL) corroborated with the findings of ORA. This example highlights the importance of new technologies in patients' evaluation with suspected glaucoma, and highlights that the correction of TAG, based on linear algorithms related to central corneal thickness alone, can determine serious errors in clinical interpretation.

Keywords: Cornea/physiology; Glaucoma; ORA; Fuchs endothelial dystrophy;Tonometry, ocular/methods; Case reports

\footnotetext{
${ }^{1}$ Centro de Estudos e Pesquisas Oculistas Associados (CEPOA) - Rio de Janeiro (RJ), Brasil;

${ }^{2}$ Grupo de Estudos de Tomografia e Biomecânica de Córnea do Rio de Janeiro (RJ), Brasil;

${ }^{3}$ Instituto de Olhos Renato Ambrósio - Rio de Janeiro (RJ), Brasil.
}

Instituto de Olhos Renato Ambrósio - Rio de Janeiro (RJ), Brasil.

Os autores declaram inexistir conflitos de interesse

Recebido para publicação em 3/2/2011 - Aceito para publicação em 26/6/2011 


\section{INTRODUÇÃO}

$\mathbf{O}$ glaucoma é uma neuropatia óptica com lesões características que causam defeitos específicos no campo visual. ${ }^{(1,2)} \mathrm{O}$ aumento da pressão intra-ocular (PIO) é o fator de risco mais importante, sendo também o foco da terapêutica para controle da doença. ${ }^{(3,4)}$ Variações da estrutura corneana geram alterações nas medidas da PIO, principalmente por meio da tonometria de aplanação de Goldmann (TAG). ${ }^{(5,6)} \mathrm{Di}$ ante desse fato, a córnea tornou-se objeto de estudo nas lesões glaucomatosas já há algum tempo, principalmente com a paquimetria central, que faz a medida da espessura corneana central (ECC). A média da ECC na população é de 556 micra, segundo Ambrósio, com desvio padrão de 35 micra, variando entre 454 e 669 micra. ${ }^{7}$ A ECC foi demonstrada como fator de risco mais importante em pacientes hipertensos oculares. ${ }^{(8)}$ A explicação para isto está vinculada ao fato de que as medidas da PIO através da TAG hipoestimam as aferições em córneas com a espessura diminuída, e por isso não são tratados, ${ }^{(9,10)}$ consequentemente aumentam o risco de progressão da escavação do nervo óptico devido à constante lesão causada pela PIO aumentada.

O modelo de predição que combina os dados do OHTS (Ocular Hypertension Treatment Study) e do EGPS (European Glaucoma Prevention Study Group) calcula para cada 40 micra de diminuição da ECC, há um risco duas vezes maior de desenvolvimento de lesão glaucomatosa em cinco anos. ${ }^{9}$

Outros parâmetros da córnea, como a ceratometria, também exercem importante influência no "erro" da medida da PIO por aplanação, que é hiperestimada em casos de córneas mais espessas e mais curvas. Diante desses fatos, levantou-se o questionamento sobre a necessidade da existência de novos parâmetros para uma melhor avaliação da córnea, frente a sua fundamental importância no seguimento dos pacientes glaucomatosos.

A paquimetria é um exame fundamental na avaliação desses pacientes, porém o seu uso isolado pode trazer uma série de equívocos de interpretação nos seus resultados, tornando-se necessário o uso de novas tecnologias, como por exemplo, o aparelho ORA (Ocular Response Analyser, $®$ Reichert).

O ORA é um tonômetro de sopro que utiliza um pulso de ar extremamente controlado e monitorado em sua intensidade, com fases ascendentes e descendentes simétricas. $\mathrm{O}$ exame leva cerca de 20 milisegundos. $\mathrm{O}$ pulso de ar desencadeia deformação na superfície da córnea, que passa por um primeiro momento de aplanação, que é identificado por um pico no reflexo da córnea (Figura 1). Após tal momento, a córnea ainda sofre a pressão do pulso de ar até ficar ligeiramente côncava (Figura 1), e retorna ao seu estado original de modo a passar por um segundo momento de aplanação (Figura 1). O comportamento da córnea e o sinal obtido durante o exame representam as propriedades biomecânicas da córnea medida. A diferença entre as pressões, nos momentos de aplanação de entrada e saída, está relacionada com a capacidade viscoelástica da córnea de armazenar energia, denominada histerese.

Quatro parâmetros são gerados na versão atual do software do ORA: ${ }^{(11)}$

- PIO calibrada para Goldmann;

- PIO compensada da córnea;

- Histerese;

- Fator de resistência corneana.

É possível analisar as alterações biomecânicas sofridas, apresentando uma diminuição importante na capacidade viscoelástica da córnea para absorver energia do sopro de ar simétrico ${ }^{(11)}$ (sua fase ascendente é idêntica a sua fase descendente).

\section{Relato de caso}

Paciente do sexo feminino, 54 anos, branca, do lar, natural do Rio de Janeiro, sem comorbidades oculares pré-existentes, em tratamento para hipertensão arterial com Captopril 25mg 2x/ao dia, veio ao serviço com queixa de redução da acuidade visual em ambos os olhos (AO), principalmente em olho direito. Relato de mãe com história de glaucoma. Ao exame oftalmológico, a paciente apresentava acuidade visual sem correção de 20/150 em AO. Realizada a refração, a acuidade passou a 20/40 e 20/30 respectivamente. (refração: $+1,75=$ $\left.0,50 \times 90^{\circ}-20 / 40 /+1,50=-0,50 \times 85^{\circ}-20 / 30\right)$.

A TAG era de $18 \mathrm{mmHg}$ no olho direito e $17 \mathrm{mmHg}$ no olho esquerdo, às 17 horas. Ao exame na lâmpada de fenda (biomicroscopia) observou-se em $\mathrm{AO}$ córneas transparentes, com guttata 2+/4+ (Figura 2), catarata nuclear grau 2 e catarata subcapsular posterior leve. Na gonioscopia, ângulo aberto até o esporão escleral nos quatro quadrantes em AO. Campo visual revelando diminuição difusa da sensibilidade em AO sem alterações específicas.

Foi medida a espessura corneana central através da paquimetria, com valor de 620 micra em AO. ${ }^{(7)}$ Realizaram-se os exames de não contato e avaliação 
biomecânica com sopro de ar (ORA), tonômetro de contorno dinâmico (TCD-Pascal ${ }^{\circledR}$ Ziemer), além da tomografia ${ }^{(12,13)}$ de córnea e segmento anterior por fotografias Scheimpflug rotacionais. ${ }^{(13,14)}$ Foram apresentados os seguintes valores: Histerese $=7,9$ e $8,1 \mathrm{mmHg}$ (valor normal médio $=10,17$ ); IOPcc(PIO corrigida pela córnea)=26,1 e 24,2 mmHg; IOPG = 18,1 e 17,9 mmHg; PASCAL (TCD): 27,2 e $26,5 \mathrm{mmHg}$. O estudo tomográfico demonstrou padrão de paquimetria espacial compatível com edema subclínico. Esse padrão é sugerido quando o perfil de progressão paquimétrico é retificado ou horizontalizado (Figura 3). ${ }^{(12)}$ Retinografia simples do nervo óptico apresentava a escavação aumentada e assimétrica, $0,8 \times 0,8$ e $0,6 \times 0,6$ respectivamente no olho direito e no olho esquerdo. Mácula sem alterações e retina aplicada em todos os quadrantes.

\section{Discussão}

Desde a publicação do estudo OHTS (Ocular Hypertension Treatment Study), em 2002, sabemos que ECC é o fator preditivo mais importante para o desenvolvimento do glaucoma em pacientes hipertensos oculares. Sendo bem claro que a medida da pressão intraocular (PIO) é hiperestimada em córneas espessas, enquanto que em córneas delgadas esta pressão é hipoestimada.

Porém, córneas com aumento da espessura por edema, apresentam resistência reduzida, e, portanto um efeito na TAG similar a córneas finas..$^{(7)}$

Com isso, fica bem claro que a paquimetria continua sendo um exame de grande valor na avaliação corneana frente ao glaucoma. No entanto, seu uso isolado passa a ser questionado diante dos possíveis equívocos no diagnóstico, que prejudicam o correto acompanhamento terapêutico do paciente.

Para o tratamento e acompanhamento clínico do caso relatado, tornou-se fundamental a avaliação biomecânica e tomográfica da córnea, com o ORA e o Pentacam, que possibilitaram análise minuciosa, de um edema corneano decorrente da distrofia de Fuchs, que levou a uma PIO hipoestimada, sendo controlada e tratada corretamente.

Importante salientar que a aferição considerada definitivamente correta entre as mencionadas como TAG, ORA e PASCAL somente seria possível, se paralelamente se confirmasse a pressão na CA (câmara anterior) com um recurso tipo canalização direta dentro da CA e a medida hidrostática. Porém, por ser um método invasivo, este procedimento torna-se impraticável na rotina clínica.

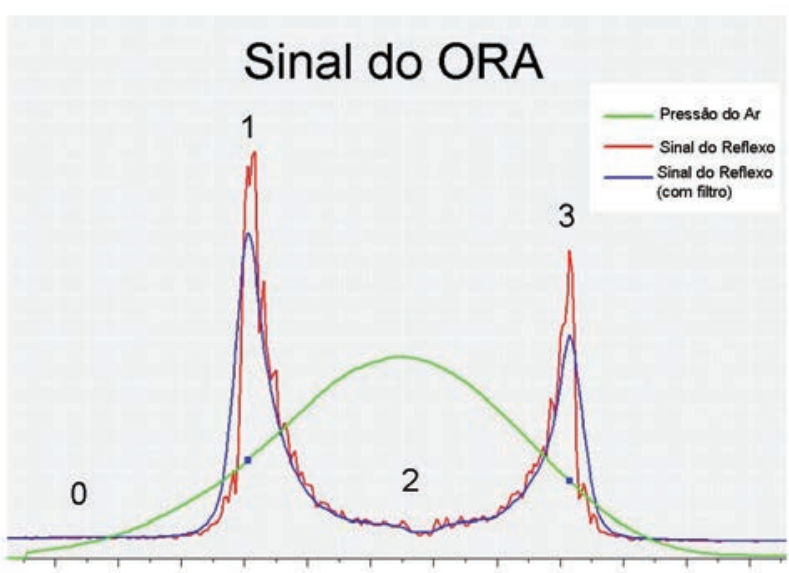

Figura 1: Sinal do ORA

. Momento 1: primeira aplanação da córnea;

- Momento 2: a córnea fica ligeiramente côncava;

. Momento 3 : segunda aplanação da córnea;

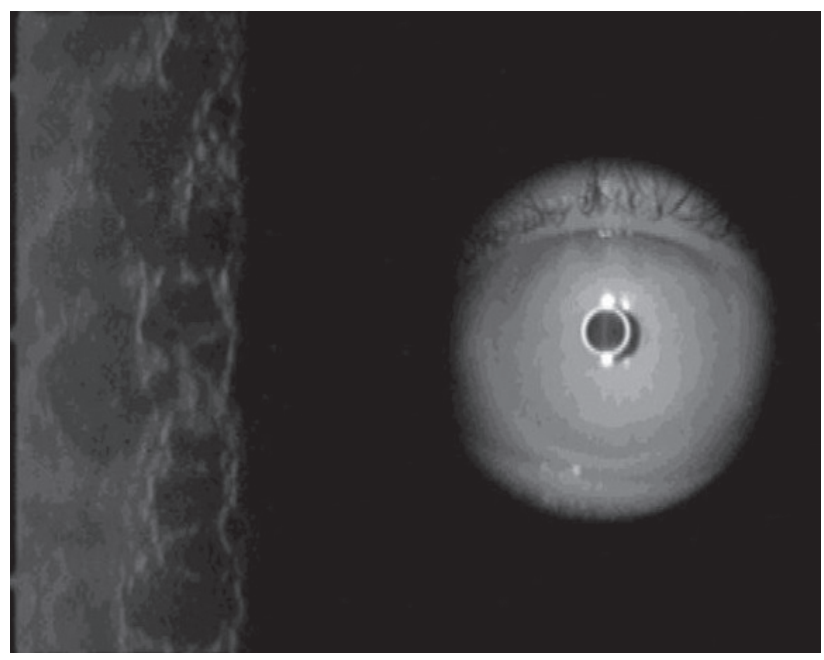

Figura 2: Microscopia especular

\section{Conclusão}

Diante deste caso clínico, fica inquestionável a influência da córnea na medida da PIO por TAG. Entretanto, ela se dá de forma mais complexa que a antecipada por algoritmos lineares considerando-se exclusivamente a paquimetria central. Neste caso, se considerássemos a TAG e a paquimetria central relativamente elevada, um grave erro de interpretação ocorreria, pois o achado de hipertensão ocular seria "mascarado". O diagnóstico de glaucoma é realizado com base nos achados do nervo óptico.

Entretanto, o estudo biomecânico e tomográfico permitiu uma correta interpretação da influência da córnea na TAG, de modo a identificar a hipertensão ocu- 


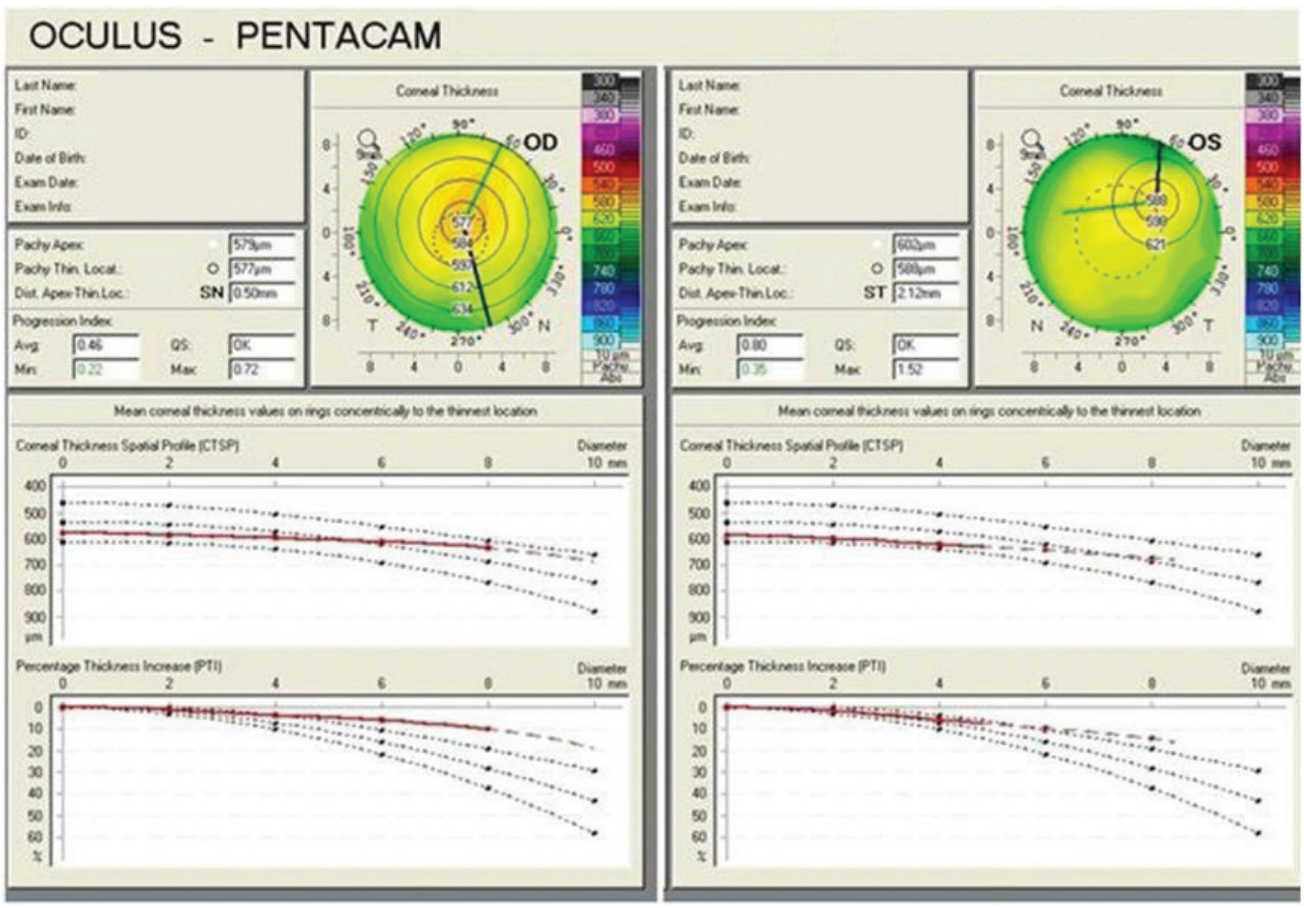

Figura 3: Curva de progressão paquimétrica espacial ${ }^{(12)} \operatorname{com}$ retificação do padrão de distribuição paquimétrico espacial

lar, e diagnosticar o glaucoma primário de ângulo aberto (GPAA). Tais exames devem ser considerados na avaliação de pacientes com suspeita de glaucoma, bem como no seu acompanhamento clínico.

\section{RefERÊNCIAS}

1. Mello PAA, Susanna Júnior R. Glaucoma. Rio de Janeiro: Cultura Médica; 2009. p.111-35. (Série Oftalmologia Brasileira).

2. Ritch R, Shields MB, Krupin T, editors. The glaucomas. 2nd ed. St. Louis: Mosby; 1996. p. 753-65.

3. Kanski JJ. Oftalmologia clínica: uma abordagem sistemática. 6a. ed. Rio de Janeiro: Elsevier; 2008. p. 372-89

4. Goldmann H, Schmidt T. [Applanation tonometry]. Ophthalmologica. 1957;134(4):221-42. German.

5. Campos M, Ambrósio Júnior R, Chamon W. Cirurgia refrativa. Rio de Janeiro: Cultura Médica; 2008. p. 136-141. (Série Oftalmologia Brasileira).

6. Luce DA. Determining in vivo biomechanical properties of the cornea with an ocular response analyzer. J Cataract Refract Surg. 2005;31(1):156-62.

7. Ambrósio R Jr, Klyce SD, Wilson SE. Corneal topographic and pachymetric screening of keratorefractive patients. J Refract Surg 2003;19(1):24-9.

8. Gordon MO, Beiser JA, Brandt JD, Heuer DK, Higginbotham EJ, Johnson CA, et al. The Ocular Hypertension Treatment Study: baseline factors that predict the onset of primary openangle glaucoma. Arch Ophthalmol. 2002;120(6):714-20; discussion 829-30.
9. Ocular Hypertension Treatment Study Group; European Glaucoma Prevention Study Group, Gordon MO, Torri V, Miglior S, Beiser JA, Floriani I, Miller JP, et al. Validated prediction model for the development of primary open-angle glaucoma in individuals with ocular hypertension. Ophthalmology. 2007;114(1):10-9.

10. Valbon BF, Guerra F, Silva RS, Canedo AL, Ambrósio Júnior R. Hipertensão ocular "mascarada" por edema de córnea após cirurgia de catarata. Rev Bras Oftalmol. 2009;68(6):34854.

11. Anand A, De Moraes CG, Teng CC, Tello C, Liebmann JM, Ritch R. Corneal hysteresis and visual field asymmetry in open angle glaucoma. Invest Ophthalmol Vis Sci. 2010;51(12):6514-8.

12. Ambrósio R Jr, Alonso RS, Luz A, Coca Velarde LG. Cornealthickness spatial profile and corneal-volume distribution: tomographic indices to detect keratoconus. J Cataract Refract Surg. 2006;32(11):1851-9.

13. Ambrósio R Jr, Belin MW. Imaging of the cornea: topography vs tomography. J Refract Surg. 2010;26(11):847-9.

14. Netto MV, Ambrósio Júnior R, Schor P, Chalita MR, Chamon W, editores. Wavefront, topografia e tomografia da córnea e segmento anterior: atualização propedêutica em cirurgia refrativa. Rio de Janeiro: Cultura Médica; 2006. p. 141-9.

\section{Endereço para correspondência Jorge Augusto Siqueira da Silva}

Rua Mem de Sá, $n^{\circ} 169$ apto. 904 - Icaraí

Niterói (RJ), Brasil

E-mail: jorgejass@ig.com.br/ jorgejass1@yahoo.com.br 